\title{
Neural Network For The Estimation Of Ammonia Concentration In Breath Of Kidney Dialysis Patients
}

\author{
Ima O. Essiet \\ Department of Electrical EngineeringBayero University, PMB 3011 Kano, Nigeria
}

\begin{abstract}
Neural networks are an extremely powerful tool for data mining. They are especially useful in cases involving data classification where it is difficult to establish a pattern in the search space. In an era when artificial intelligence is increasingly being utilised in industrial and medical applications throughout the world, it is becoming evident that this is an emerging trend. This paper explores the idea of artificial intelligence by employing the use of a feed-forward neural network with two process layers to determine the concentration of ammonia in exhaled human breath. The human mouth contains many kinds of substances both in liquid and gaseous form. The individual concentrations of each of these substances could provide useful insight to the health condition of the entire body. Ammonia is one of such substances whose concentration in the mouth has revealed the presence or absence of diseases in the body. Kidney failure is one diesease which is identified by an extremely high ammonia content in human breath. This disease is as a result of the kidneys' inability to process the body's liquid waste. The result of this is the release of urea throughout the body which is dissipated in the form of ammonia through oral breath. The neural simulation is carried out using NeuroSolutions version 5 software. The neural network correctly identified the concentration of oral ammonia as an indication of kidney failure with an accuracy of $85 \%$.
\end{abstract}

Keywords: ammonia, BUN, kidney failure, metal oxide semiconductor, NeuroSolutions, neural network

\section{Introduction}

Artificial neural networks are information processing models which function very closely to the way the human brain does. They consist of a number of interconnected processing elements called neurons. These neurons are connected to other neurons via a structure called a dendrite. The dendrites receive information from other neurons via axons. At the end of a dendrite is a synapse which converts axon activity into electrical signals which inhibit or excite activity in the connected neurons. When a neuron receives excitatory input that is sufficiently large compared with its inhibitory input, it sends a spike of electrical activity down its axon. Learning occurs by changing the effectiveness of the synapses so that the influence of one neuron on another changes (Papert, 1969). Neural networks mostly learn by example and do so through either supervised or unsupervised means. Artificial neural networks are particularly useful in data classification and pattern recognition. Other properties of neural networks that make them suitable for use in artificial intelligence include generalization, graceful degradation, adaptation, and parallelism (Graupe, 2007). The simplest form of an artificial neural network is the perceptron. An illustration of a perceptron is shown in figure 1.

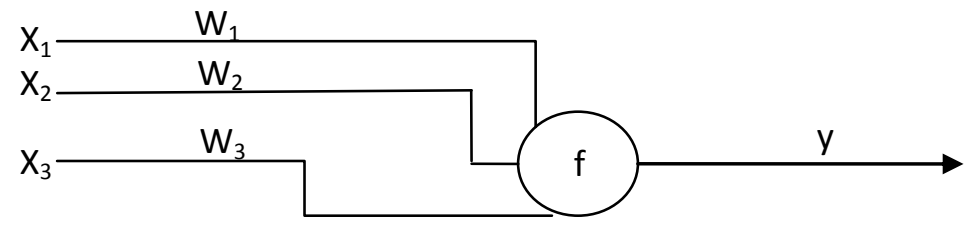

Figure 1. A Simple Perceptron with three inputs, associated weights and one output

Feed-forward neural networks allow a signal to travel in only one direction i.e. from input to output. There is no form of feedback associated with this type of neural network architecture. They are mostly suitable for pattern recognition purposes. There are basically three layers of information processing in a neural network. The first is called the input layer which accepts the information to be processed. The next is the hidden or process layer which acts on the information supplied by the input layer along with weights connecting the input and hidden layers. The output layer supplies the results of the process layer along with the weights connecting the hidden and output layers. A neural network with one hidden layer is called a single-layer perceptron, while one with more than one process layer is called a multi-layer perceptron (MLP). For simple cases involving pattern recognition, a single-layer perceptron would suffice. However, for more complex cases of data classification, a multi-layer perceptron would give more accurate results (Svozil, D., Kvasnicka, V., Pospichal, J., 1997).

Various learning methods are available for training neural networks. The two most commonly used are supervised learning and unsupervised learning. In supervised learning, desired outputs are already known for 
associated input patterns. Whenever an input is applied to the neural network, the network's parameters are adjusted according to the difference between the desired and actual output of the neural network. Supervised learning methods include error-correction learning, reinforcement learning and stochastic learning. The aim of supervised learning is to determine a set of weights that minimises the error between the actual and desired outputs. A commonly used method is the least mean square (LMS) convergence method (Alkon, 1989).

The ammonia odour in the mouth of kidney failure patients is associated with high levels of blood urea nitrogen(BUN). When the kidneys are not functioning well, blood urea nitrogen will accumulate in the body. The excess urea will be decomposed into ammonia under the effect of urea enzymes in the gastrointestinal tract. Ammonia will be discharged through the oral cavity(mouth) to cause ammonia smells in the mouth. Also, high BUN in saliva can also be decomposed into ammonia to make the ammonia odour in the mouth worse.

Haemodialysis is a treatment that involves the use of specially designed machines to reduce the BUN levels in patients who suffer from kidney failure. This due to the fact that diseased kidneys can no longer filter the blood's urea-nitrogen. As a result, there is a build up of these compounds in the body to the point that it becomes toxic. At this point, patients are said to suffer from kidney failure. Although analysis of body fluids (blood, sputum,urine) for disease diagnosis and monitoring is routine clinical practice, human breath analysis methodologies that exploit the non-invasive nature of such an approach are still under-developed. Since the time of the ancient physician Hippocrates, exhaled breath was recognized as a non-invasive tool to diagnose diseases. Breath testing devices first appeared in 1784 when Lavoisier detected CO in exhaled breath of guinea pigs [1]. Since then, colorimetric assays and gas chromatography columns have been used to analyze VOCs (volatile organic compounds) in human breath in quantities varying from millimolar $\left(10^{-3} \mathrm{M}\right)$ to picomolar $\left(10^{-12} \mathrm{M}\right)$ concentrations [1].

\section{Theoretical Background}

Many forms of the feedforward multi-layer perceptron(MLP) have been used in the past which yielded encouraging results. These have been applied in different fields such as in chemistry ( Svozil, D., Kvasnicka, V., Pospichal, J., 1997), food industry (Balasubramanian, S., Panigrahi, S., et al, 2009), medicine (Kodogiannis, V.S., Lygouras, J.N. et al, 2008), and agriculture (Kizil, U., Lindley, J.A., 2009), to mention a few. A multilayer perceptron as the name implies, is a neural network that consists of multiple layers of neurons which exchange information. While there are many forms of the MLP, the simplest is one with three layers: input layer, hidden(process) layer and output layer. Figure 2 illustrates the architecture of a feedforward three-layer MLP.

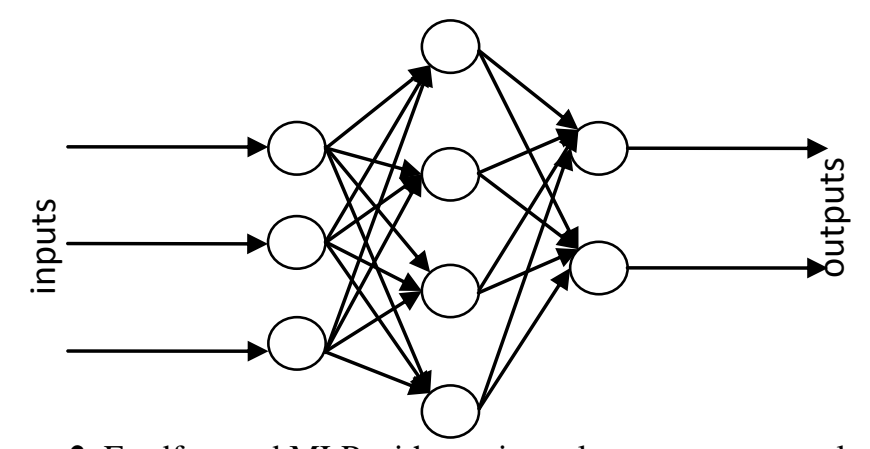

Figure 2. Feedforward MLP with one input layer, one process layer and one output layer

There is no rule-of-thumb guiding the selection of a particular neural network model for a specific application. However, there are factors that can provide insight concerning the model that may yield the best results. Some of these include the number of training cases, the amount of noise, the complexity of the function or classification being learned, and also the method of training (Hagan, M.T., Menhaj, M.B., 1994). There are important aspects of neural network training which must be considered if the network is to yield useful results. These include:

(i) Transfer function: two functions determine the way signals are processed by neurons. The first is the activation function which determines the total signal a neuron receives. For a neuron $a$ connected to a set of neurons $b$ (for $\mathrm{b}=1 \ldots \ldots \ldots . \mathrm{N})$, sending signals $n_{b}$ with the strength of the connections being $W_{a b}$, the total activation $A_{a}(b)$ is given as:

$A_{a}(b)=\sum_{b=1}^{N} W_{a b} n_{b}$

The second function determining the network's processing capability is the output function. The combination of both the activation and output functions determine the transfer function. 
(ii) Interconnected Weights: these exist between the various layers of artificial neurons and are used in combination with the transfer function to vary the signals that each neuron receives. Assume two interconnected neurons $a$ and $b$ exchange information through a common axon. The weight associated with both neurons is given as $W_{a b}$ such that $\left\{W_{a b} \in 0 \ldots . .1\right\}$. The closer the value of $W_{a b}$ is to 1 , the greater the importance of the associated connection.

(iii) Neuron (node) Biasing: this is a real-valued factor that is used to vary the internal processing operations of a particular node within the network. It is also selected in combination with the transfer function.

Supervised learning is a method of training neural networks to automatically perform tasks whose outcome(s) is/are known. One common use of supervised learning is in data classification. The trainer is aware of the correct(desired) results of the classification process. The training process will therefore involve the continuous minimization of the error between the actual outputs of the neural network $\left(O A_{n}\right)$ and the desired outputs $\left(O D_{n}\right)$. The resulting error is given as:

$$
\left\{E_{n}\right\}=\left\{O A_{n}-O D_{n}\right\}
$$

The supervised learning paradigm of training neural networks is efficient and offers solutions to several linear and non-linear problems such as classification, plant control, forecasting, prediction and robotics (Sathya, R., Abraham, A., 2013). The two most common forms of supervised learning include error-correction learning and memory-based learning. The method used in this work is the error back-propagation method which is a form of error-correction.

The error back-propagation method involves adjusting network weights in such a way that the error between the actual and desired outputs is minimized as much as possible (Rumelhart, Hinton and Williams, 1986). This is done by computing the error derivatives of the associated weights. These error dervatives are obtained by error back-propagation. In error back-propagation, the steepest descent minimization method is used ( Svozil, D., Kvasnicka, V., Pospichal, J., 1997). Assume we intend to adjust the weight between two neurons $a$ and $b$. Weight and threshold coefficents can be adjusted according to the following:

$$
\begin{aligned}
& w_{a b}^{k+1}=w_{a b}^{k}-\eta\left(\frac{\partial E}{\partial w_{a b}}\right)^{k} \\
& v_{a}^{k+1}=v_{a}^{k}-\eta\left(\frac{\partial E}{\partial v_{a}}\right)^{k}
\end{aligned}
$$

Where $\eta$ is the learning rate $(\eta>0)$

\section{Neural Network Design And Simulation}

The neural network to be designed is to be used for the estimation of varying concentrations of ammonia in human breath for the diagnosis of kidney failure. Ammonia $\left(\mathrm{NH}_{3}\right)$ is one of the compounds whose increasing concentration in the human mouthcan be an indication of the onset of kidney failure []. In other words, the response of various kinds of gas sensors was analysed using the neural network to determine which one was best suited for measuring oral ammonia in varying concentrations. The sensors being testedhave an ammonia sensitivity in the range of parts per million ( $\mathrm{ppm})$. NeuroSolutions version 5 was used as the neural network training software.

The structure of the neural network used is a multilayer perceptron feedforward network with 3 inputs and 2 outputs. The inputs and outputs are normalized values of both kidney failure and non-kidney failure breath samples obtained by using a reference of $100 \mathrm{ppm}$ and $50 \mathrm{ppm}$ ammonia concentration for kidney failure and non-kidney failure samples respectively. These values were obtained according to the following relations:

$$
X_{g}=\frac{\text { Measured ammonia concentration for non - kidney failure sample }(\mathrm{ppm})}{50 \mathrm{ppm}}
$$

and

$$
X_{b}=\frac{\text { Measured ammonia concentration for kidney failure sample }(\mathrm{ppm})}{100 \mathrm{ppm}}
$$

Sigmoid transfer function is used in the hidden layer neurons and the learning is via error back-propagation (EBP) algorithm according to the following expression:

$$
E_{12}=\left(t_{12}-a_{12}\right) \cdot a_{12} \cdot\left(1-a_{12}\right)
$$


Where $E_{12}$ is the associated error for output nodes 1 and 2 respectively, $t_{12}$ is the target activation for nodes 1 and 2 (desired outputs), $a_{12}$ is the activation function for output nodes 1 and 2 respectively. The resulting error is then used to obtain process layer errors using the following relation:

$$
E_{k}=a_{i}\left(1-a_{k}\right) \cdot \sum_{j} E_{j} w_{i j}
$$

Where $E_{k}$ is error for process node $i, a_{k}$ is activation for node $k, E_{j}$ is output error of node $j$, and $w_{i j}$ is weight connection between nodes $i$ and $j$.

Due to the fact that the gas sensor's desirable performance is vital to ensuring that the sensor circuit gives accurate results, there is a need to simulate the chosen sensor's response to molecules of ammonia gas. The training cycle was selected as 1000 epochs which provided adequate time for the ANN to adjust to changes in associated synaptic weights. The weights between a hidden node $i$ and output node jare adjusted according to the following expression:

new $w_{i j}=$ old $w_{i j}+\eta \cdot E_{k} \cdot x_{i}$

Where $k=1,2$ and $j=1,2, \ldots 4$ for each set of 100 sample test points, $w_{i j}$ is the weight between nodes $i$ and $j$, $E_{\kappa}$ is error term calculated for output node $j, x_{i}$ is the output of hidden node $j$ and $\eta$ is a constant value called the learning rate. The ANN has four hidden nodes. For the simulations carried out the learning rate was fixed at $\eta=0.01$.

The neural network was used for data classification whereby the sensor's response to ammonia concentration in kidney failure and non-kidney failure breath samples formed the target values for training the neural network using supervised training. Non-kidney failure samples have an ammonia concentration of between 0 and 50ppm. Kidney failure samples have concentrations of 100ppm and above (Persaud, K.C., Qutob, A.A. et al, 1994). Two separate data sets were obtained from testing various samples of kidney failureand non-kidney failure samples with each set containing 100 test points. Each set of 100 test points consists of sample measurements being taken using impedance resonance method (Yim, S., Kibbey, C.E. et al, 1993). These two sets of test points were used for the neural network training sessions with a normalized standard of 0 to 0.5 for non-kidney failure breath samples and 0.6 to 1.0 for kidney failure samples respectively.

\section{Neural Network Simulation Results}

The neural network was then tested with 30 sample points from each data set to reveal the simulation results shown in Figures 3 and 4. The average cost is a metric which the neural network training software uses to measure the accuracy of the predicted data points in relation to the actual or desired values. In other words, it is a measure of how well the training process converged. The cost $(\mathrm{T})$ and cost $(\mathrm{CV})$ are both part of this average cost metric with the first applying to the training data set and the second to the cross validation data set. For a successful training session, both must converge to zero or near zero.

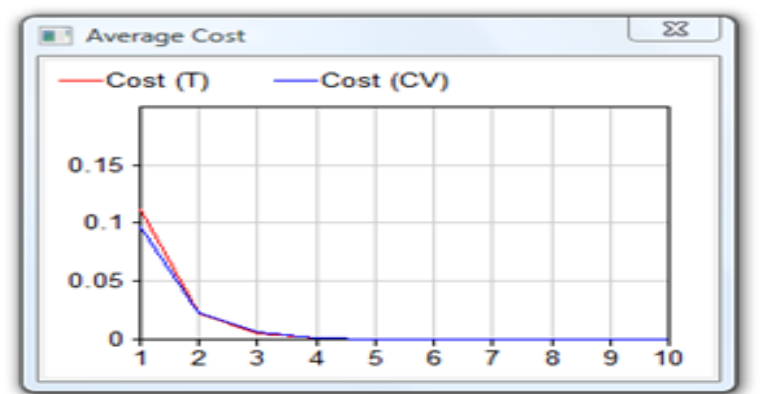

Figure 3.NeuroSolutions testing window showing convergence rate for non-kidney failure breath sample

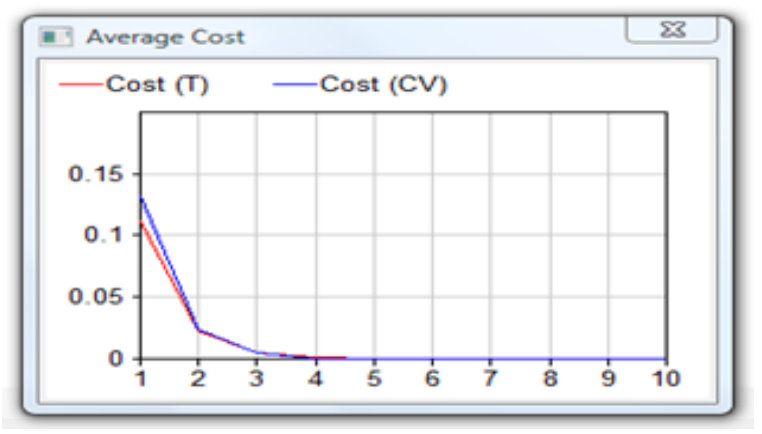


Figure 4.NeuroSolutions testing window showing convergence rate for kidney failure breath sample One of the strengths of the neural network approach to data mining is its ability to function in data spaces where the statistical distribution of the data is partly known. As a result of this, a confusion matrix has been included in Table1 to show the accuracy of the correctly predicted data points in relation to the actual number of tested data points for both kidney failure and non-kidney failure breath samples.

Table 1.Confusion matrix showing accuracy of results obtained for ammonia breath test samples

\begin{tabular}{|c|c|c|c|}
\hline & $\begin{array}{c}\text { Kidney } \\
\text { failure } \\
\text { (predicted) }\end{array}$ & $\begin{array}{c}\text { Non kidney } \\
\text { failure } \\
\text { (predicted) }\end{array}$ & $\begin{array}{c}\text { Accuracy } \\
\%\end{array}$ \\
\hline $\begin{array}{c}\text { Kidney } \\
\text { failure } \\
\text { (actual) }\end{array}$ & 9 & 1 & 90.0 \\
\hline $\begin{array}{c}\text { Non } \\
\text { kidney } \\
\text { failure } \\
\text { (actual) }\end{array}$ & 2 & 8 & 80.0 \\
\hline $\begin{array}{c}\text { Overall } \\
\text { Accuracy }\end{array}$ & & & 85.0 \\
\hline
\end{tabular}

From the confusion matrices above, it can be seen that the neural network has $85 \%$ overall average accuracy in distinguishing between kidney failure and non-kidney failurepatients. The sensor that produced these results is TGS2602 metal-oxide semiconductor gas sensor made by Figaro, Japan. This sensor was then used to implement a simple and affordable electronic device (also called an electronic nose or e-nose) which is capable of monitoring the condition of the breath samples in realtime.

\section{Conclusion}

Oral ammonia concentrations of $60-120$ ppm were observed for candidates with kidney failure. Candidates with less severe cases of kidney failure had lower breath ammonia concentrations compared to those with advanced symptoms. Candidates without kidney failure had half the concentration of oral ammonia compared to those with the disease. Also, it was observed that oral ammonia concentrations gradually decreased over the 10 second exhalation interval for all candidates tested. The results obtained using the proposed neural network show that breath ammonia analysis is an effective and accurate method of diagnosing kidney failure. It is therefore recommended that breath ammonia analysis be used by medical practitioners for the diagnosis of kidney failure.

\section{References}

[1]. Svozil, D., Kvasnicka, V., Pospichal, J. (June 6, 1997). Introduction to multi-layer feed-forward neural networks. Chemometrics and Intelligent Laboratory Systems , pp. 43-62.

[2]. Alkon, D. (1989). Memory Storage and Neural Systems. Chicago: Scientific American.

[3]. Balasubramanian, S., Panigrahi, S., et al. (2009). Neural Network-integrated Metal Oxide-based Artificial Olfactory System for Meat Spoillage Identification. Journal of Food Engineering , 91-98.

[4]. Graupe, D. (2007). Principles of Artificial Neural Networks. UK: World Scientific Publishing Co.

[5]. Hagan, M.T., Menhaj, M.B. (1994). Training Feed Forward Networks with the Marquardt Algorithm. IEEE Transactions on Neural Networks, 989-993.

[6]. Kizil, U., Lindley, J.A. (2009). Potential Use of Gas Sensors in Beef Manure Nutrient Content Estimations. African Journal of Biotechnology, 2790-2795.

[7]. Kodogiannis, V.S., Lygouras, J.N. et al. (February 16, 2008). Artificial Odor Discrimination System Using Electronic Nose and Neural Networks for the Identification of Urinary Tract Infections. IEEE Transactions on Information Technology in Biomedicine, pp. 707-708.

[8]. Papert, M. a. (1969). Perceptrons: An Introduction to Computational Geometry. Massachusetts: MIT Press.

[9]. Persaud, K.C., Qutob, A.A. et al. (1994). Odor Evaluation of Foods Using Conducting Polymer Arrays and Neural Network Pattern Recognition . In Olfaction and Taste (pp. 708-710). Tokyo: Springer-Verlag.

[10]. Rumelhart, Hinton and Williams. (1986). Learning Internal Representations by Error Propagation. Glasgow.

[11]. Sathya, R., Abraham, A. (2013). Comparison of Supervised and Unsupervised Learning Algorithms for Pattern Classification . International Journal of Advanced Research in Artificial Intelligence, 34-38.

[12]. Svozil, D., Kvasnicka, V., Pospichal, J. (1997).

[13]. Yim, S., Kibbey, C.E. et al. (August 13, 1993). Polymer Membrane-based Ion-, Gas- and Bio-Selective Potentiometric Sensors. Biosensors and Bioelectron, pp. 1-38. 